\title{
A new marine station on the Baltic
}

\author{
K. E. Skora \\ Hel Marine Station, University of Gdansk; 84-150 Hel, P.O.B. 37, Ul Morska 9, Poland
}

The Hel Marine Laboratory was established in 1977 as a preliminary field station of Gdansk University Department of Oceanography. It was the only institution of its kind in Poland, and constitutes the beginnings of Gdansk University's future marine station to be established to conduct field research and teaching in oceanographical subjects.

On 25 th September 1992, the new building was officially inaugurated as the Hel Marine Station of Gdansk University.

The location of the station in the central part of the Gulf of Gdansk makes it possible to conduct investigations in the open sea and its coastal zone, and to observe the physicochemical, biological, and geological processes taking place there, in both the water and the zone of its contact with the seabed, the coast and the atmosphere. The primary function of the station is, and will continue to be, the education of specialists in the field of basic marine ecology, with particular emphasis on rational utilization of the sea, its resources, and protection against the effects of pollution. This function is expressed in the topics discussed in class, in the special and thesis-related practical training courses, and in the work for MSc and $\mathrm{PhD}$ theses by students of Gdansk and other Universities.

The Marine Station at $\mathrm{Hel}$ is also a field back-up facility for developing all kinds of oceanographical research (fundamental, utilitarian and methodological) resulting from the statutory requirements of the Department of Oceanography and Gdansk University.

The research activities concentrate on the following:

- carrying out our own investigations leading to doctoral and postdoctoral degrees by members of the Department of Oceanography of Gdansk and other Universities,

- carrying out research projects on request, connected with the exploitation and conservation of marine resources,

- carrying out research projects within the framework of cooperation agreements, the results of which are of interest to other scientific institutions,

- agencies of the maritime economy, or regional authorities.

At present, the Hel Marine Station (HMS) trains specialists in ecology, biology, and marine environmental protection, as part of its training programme for Oceanography students. Every year, field classes are held here for about 30 third- and fourth-year students in biological oceanography. After the third and fourth year, about 10-15 students of biological oceanography and biology from Gdansk and other Polish Universities have their professional training courses here. The students learn the basics of field research work, material collection, data analysis, and evaluation of results. Work carried out by the students is usually part of more extensive research dealing in current topics. Work related to theses is also conducted. 
The Station is also prepared to organize special educational courses on natural science according to curricula prepared on request. These may, for instance, be courses for employers of the state or local administration engaged in solving problems of protecting man's natural environment.

Thanks to the opening of new laboratories and living accommodation, which will increase both the educational and research potential, scientists interested in research in this part of the part of the Baltic Sea will now be able to extend their scope of work by planning new topics. The laboratory has the infrastructure and the requirements to organize meetings for specialists from related fields.

In addition to teaching, the scientific staff of the Station carry out their own individual research, comprising the following fields:

- shallow-water fish ecology in the Baltic

- studies on biology and ecology of the Antarctic (The scientists of Hel Marine Station have participated in six expeditions)

- collection of data on the occurrence of marine mammals in the Polish zone of the Baltic. (Study on the disappearance of the harbour porpoise is of special importance here)

- a new problem, to which the HMS devotes considerable attention, is the investigation of bottom fauna in the area of discharge sites of sewage-processing plants in order to measure their effectivity as well as their impact on near-shore ecosystems

- constant monitoring of biological life of in-shore waters. The station intends taking part in the permanent monitoring of bottom macrofauna and ichthyofauna resources.

The Station is equipped with a steady-flow seawater aquarium system and thermostat rooms, making it possible to plan research projects involving rearing experiments, viz. impact of chemical pollutants on fish spawning; feeding experiments with various species; culture of live food organisms; larval studies, and new mariculture techniques.

Besides training of specialists and studies of the marine environment, the Station has been engaged in pro-ecological educational activities among school children, and it publishes a pro-ecological paper for the inhabitants of the town of Hel. Preparations are underway for making popular science films on nature.

The HMS was established to meet current social demands for ecological studies of the sea, especially in the Gulf of Gdansk and Puck Bay and to produce well-trained specialists in ecology for the protection of the marine environment. In order to fully utilize the teaching, scientific and organizational potential, the staff of the Hel Marine Station together with the authorities of Gdansk University are urgently looking for partners for teaching and scientific cooperation.

Help in this respect, as well as the carrying out of joint research and teaching ventures would no doubt favour the further development of this young institution. 